\title{
Image Analysis of Newborn Plantar Surface for Gestational Age Determination
}

\author{
Olga R.P. Bellon ${ }^{\star 1}$, Maurício Severich ${ }^{1}$, Luciano Silva ${ }^{2}$, Mônica N.L. Cat ${ }^{3}$, and \\ Kim L. Boyer ${ }^{4}$ \\ 1 Universidade Federal do Paraná - Dept. Informática, Curitiba/PR-Brasil \\ \{olga, luciano, mseveric\}@inf.ufpr.br, \\ 2 Centro Federal de Educação Tecnológica - CPGEI, Curitiba-PR-Brasil \\ 3 Universidade Federal do Paraná - Hospital de Clínicas, Curitiba/PR-Brasil \\ monica@hc.ufpr.br \\ 4 The Ohio State University - Dept. Electrical Engineering, Columbus/OH-USA \\ kim@ee.eng.ohio-state.edu
}

\begin{abstract}
We present a computational approach to support gestational age determination of premature newborns. This knowledge is fundamental to guide postnatal treatment and increase survival chances. However, current approaches are both invasive and do not generate precise results. This paper presents an original and non-invasive method to determine the gestational age based on information supplied by plantar surface images. These images present many details and patterns but to date have not received attention from the image processing community. We provide a computational tool with suitable facilities to allow the image analysis, either automatically or user-driven. Besides its importance to Neonatology, this work represents a contribution of Computer Vision concepts to Medical Imaging. The experimental results confirm the relationship between the plantar surface features and newborn gestational age.
\end{abstract}

\section{Introduction}

Medical images supply important information in patient diagnoses and treatments. Several applications in Medicine have benefited from different image processing techniques, such as organ segmentation and visualization [1. This paper presents an original contribution to medical imaging in the use of image processing techniques in estimating the gestational age of premature newborns.

The gestational age is fundamental to guide treatment of newborns and is defined according to his/her physiological development. Often, the estimated gestational age determines the feasibility of the treatment. Presently, there are suitable methods to determine the gestational age, but they depend on adequate prenatal attendance and ultrasonic evaluation, achieved in the first trimester of gestation [2]. In many situations, for a variety of reasons [3], the needed information is not available. The alternative is to adopt different postnatal evaluation

\footnotetext{
* The authors, L. Silva and O.R.P. Bellon thank to CAPES for financial support.
} 
methods [4], which have three disadvantages: 1) they are invasive - requiring intensive newborn handling; 2) they are imprecise - are not based on premature populations; and 3) they require high levels of expertise in Neonatology.

Studies performed by Neonatologists show that the problems suffered by premature newborns differ from those in full term babies. There is a great difference in survival probabilities and neurological outcome, especially between 24.5 and 25.5 weeks [5], Fig. 1] Furthermore, technological evolution has provided new clinical possibilities, increasing the success rate in premature newborn treatment [2]. Therefore, knowledge of gestational age has become very important and it is evident that a non-invasive and more precise method of determining this age is needed. We propose a new approach, FootScanAge, to determine gestational age by analyzing the plantar surface image.

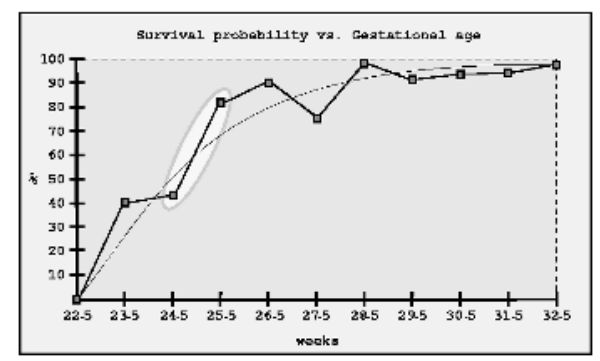

Fig. 1. Survival probability (\%) vs. Gestational age in weeks.

The FootScanAge method is based on the observation that both the plantar surface and plantar wrinkles present different shapes and follow reasonably predictable patterns according to the gestational age. Moreover, it is known that wrinkles in the plantar surface start to develop when the fetus has 18 weeks [6]. We developed a prototype of an information system with the goal of validating this hypothesis. This paper presents the image-processing tool of the system, designed to provide information required by the method, which includes: 1) size of the plantar surface; 2) area and shape of the plantar surface; 3) characteristics of plantar wrinkles; and 4) percentage of wrinkle occupancy. We also present experimental results confirming some initial assumptions about the method.

The plantar surface image is unusual and presents details that must be preserved during its processing. The main problem is the high variation of wrinkle patterns. We tested several approaches to image acquisition: 1) hand scanning, presenting difficulties in handling on the fragile plantar region of premature newborns; 2) digital photography, which presents difficulties in standardizing the position of plantar surface and the illumination conditions; and 3) table scanning for plantar impression on a sheet, usually made in the delivery room just after the birth for record keeping, which underscored the poor quality of the currently achieved images. Despite these difficulties, the plantar impression generated the 
most promising type of image. But it was necessary to re-evaluate the procedure to acquire the impressions, in light of our requirements. This led to a review of technical problems in the acquisition of newborn registration images. Following suitable modifications, the plantar impression produces images considered satisfactory for computational processing and is used in our system (Fig. 2(a).

\section{The Image-Processing Tool}

Although an ideal solution would be autonomous in medical applications, user intervention is almost always required due to domain complexity and image acquisition limitations 7]. Our image processing tool was designed based on this concept and comprises four stages, as discussed below, providing facilities to help the expert in the analysis of plantar surfaces. The tool allows the user to supervise and modify the tuning parameters of each stage, improving the automatic processing results. These parameters are stored in a database and the most used are automatically selected as default values for subsequent analyses.

\subsection{Region of Interest (ROI) Extraction}

This stage identifies the image background, (Fig. 2(c)), and its four main areas (Fig. 2(d) , which are individually analyzed during the measurement stage. The first step is a global binarization using an automatic threshold T. Our approach to setting $T$ is based on the literature 89] and uses two measures of central tendency and three measures of variation (Eq. 1), where: $\bar{X}$, is the average value of all image pixels, $A D e v$ is the image mean absolute deviation, Med is the image median value, $M D e v$ is the image median absolute deviation, and $\sigma$ is the image standard deviation.

$$
T=((\bar{X}+A D e v+M e d+M D e v) / 2)-\sigma
$$

Our experimental results show that for plantar surface images our threshold technique works better than when only the mean or median value is used. This approach also outperforms the well-known Otsu's threshold [10]. The binary image is processed to correct the foot orientation, facilitating the extraction of measures. Then, a morphological closing method [11] is applied to the image (Fig. 2(b) to fill small gaps, allowing the identification of the plantar surface boundaries. The user selects the number of closing iterations to achieve better results in specific situations (Fig. 2(c)). The closed image is partitioned into four regions (Fig. 2(d) by using the known length percentage of each region, adapted from [6]. The objective is both to allow the computation of ratios between different areas and to verify the behavior of wrinkles in each area, according to the needs of the FootScanAge method.

\subsection{Edge-Preserving Noise/Speckle Reduction}

Because we seek to analyze the wrinkles of the plantar surface, we searched for an enhancement technique that would improve the image quality without corrupting 


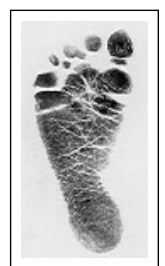

(a)

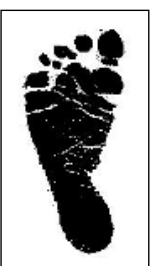

(b)

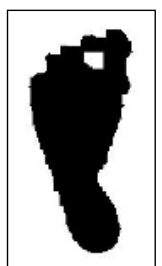

(c)

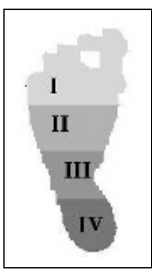

(d)

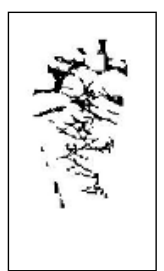

(e)

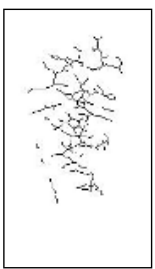

(f)

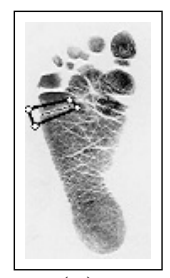

(g)

Fig. 2. Different stages: (a) Plantar surface image; (b) Binary image; (c) Plantar region; (d) Four main regions; (e) Wrinkles; (f) Thinned wrinkles; (g) User selection.

fine details is justified. Thus, 186 plantar surface images were used to evaluate a variety of filters. By comparing of well-known common filters, such as Mean and Median, against edge-preserving noise reduction filters, such as SensiEdge [12], NGIW (New Gradient Inverse Weighted Filter) [13] and DGIW (Directional Gradient Inverse Weighted Filter) [14, we see that the edge-preserving filtering best preserves the wrinkle shapes as needed.

SensiEdge 12] detects an edge and its orientation in a local neighborhood. A pre-specified threshold $t_{1}$ is used to identify edge pixels and the selected pixels are used in Mean filtering. Our modified SensiEdge improves the previous method by using local gradient information to automatically define edge pixels and by using Median filtering, which better preserves edges in this specific situation.

Although the DGIW yields more homogeneous regions, the masks used in selecting the optimal neighborhood affect its performance. Hence some image structures that should be preserved can be suppressed. The DGIW selects as optimum the neighborhood with minimum variance; this is a valuable approach in the presence of impulsive noise. Otherwise, it can use the current pixel value rather than the neighborhood mean to compute all neighborhood variances.

Since the DGIW uses the filter response of the selected neighborhood, and neighborhoods are set by square masks [14, this operation can generate chessboard artifacts in some image structures. It was observed in our tests that chessboard artifacts are more pronounced by using the NGIW [13]. To minimize this, the user can select Median instead of the NGIW. However, the Median can obliterate thin lines, and thus it should only be used under known conditions.

For noise reduction, we also developed an adaptive edge-preserving filter combining local statistics, especially designed line masks [11, Median filter, our modified DGIW and the NGIW. This filter provided the best results of all filters studied. Local statistics are used to estimate: 1) the edge pixel candidates, and 2) the most suitable filter to apply. Depending on this estimate, the filter computes the median of the selected line mask or applies the DGIW or the NGIW. Due to the relative complexity in filter adjustment, from the user's point of view (Neonatologists), we reduced the filtering options to only those with the best results: the NGIW, our modified SensiEdge, and the developed edge-preserving filter. 


\subsection{Wrinkle Detection}

The plantar wrinkles are detected by region growing, which uses 1) the Niblack adaptive thresholding [9] to select seeds, and 2) a local threshold with region statistical information to assign a plantar wrinkle label to a pixel. Initially, it is verified if a pixel can be a seed of a wrinkle region: if its value is greater than the Niblack threshold $t_{2}$, within an $\mathrm{NxN}$ window, then it can be a seed, $s_{i}$. A new region is then initialized and a growing process, using 8-connectivity, is started from $s_{i}$. In our tests, values of $\mathrm{N}$ from 40 to 50 were effective.

A pixel can be joined to a region if it satisfies a homogeneity criterion $p$, $[m-t<p<m+t]$, where $m$ is the region mean value and $t$ is a tolerance that depends on the number of pixels of the region. If a region has less than $n$ pixels, $t$ is the user suggested tolerance. Otherwise, $t$ is the region absolute mean deviation $d$. In our experiments, we set $n$ between 10 and 25, as lower values do not produce reliable absolute mean deviation and higher values tend to favor the user suggested tolerance.

The detected wrinkles, (Fig. 2(e), are shown to the user for validation or refinement of the detection parameters. Then, a thinning process is applied to obtain the skeleton of the wrinkles, (Fig. 2(f) . In this process, a morphological thinning algorithm was performed based on the distance transform and structuring elements of Mathematical Morphology [15. The line crosses and forks on the skeleton (Fig. 2(f) are computed and a set of vertices $v$ are defined. This information is used to remove undesirable parasitical lines from the skeleton.

\subsection{Image Measurements}

To standardize the measurements, the resolution of the acquisition device was fixed to $200 \mathrm{dpi}$. Thus, the measurements provided to the user are directly mapped using this information. Some measurements are automatically computed and presented to the user, such as plantar surface measurements (length, width, area, occupation) and regions measurements (area and "corrugation").

The initial image (Fig. 2(a) is shown to the user who then defines a region in which wrinkles must be measured (Fig. 2(g) . The region vertices are set by interactive "mouse clicks" on the image. The system computes the wrinkle measurements for all wrinkles detected within the selected area. This process must be done to all desired wrinkles. The measured lengths are the number of pixels in the selected lines. The measured widths are computed as the median value of the wrinkle skeleton values previously calculated by the thinning algorithm.

\section{Experimental Results}

\subsection{Image Processing}

The current literature reveals no work on plantar surface image analysis, so a direct comparison to prior work can not be performed, but a visual evaluation is presented in this section. As described, the image-processing tool was developed 
on a user-driven basis. However, as a quantitative experiment, 186 images were processed without user intervention to observe the tool behavior in performing different tasks.

ROI extraction: Although our automatic threshold yields better foot segmentation than Otsu's approach (Fig. 3) the tool failed to identify the plantar surface in 5 images. This occurred either due to the use of an inaccurate automatic threshold, or not enough morphological closing iterations, set to 13 iterations in the initial experiments.

Noise reduction: Our adaptive edge-preserving filter was chosen to process all images. The filter has a single parameter that defines the number of iterations and was set to two, yielding satisfactory results. The filter behavior tends to be stable with more than two iterations, generating only minor changes. From visual comparison, the NGIW was chosen (three iterations), because it yielded the best results in all experiments. Some image structures, such as thin lines and line endings, are blurred by NGIW filter (Fig. 4).

Wrinkle detection: The initial parameter to adjust wrinkle detection is the user tolerance, which was not based on user visual judgment but on the absolute mean deviation of the image. This solution was not suitable for wrinkle detection on some images, since only a little amount of wrinkle were identified (Fig. 5(b), although wrinkle detection was satisfactory on most images (Fig. 5(e)].

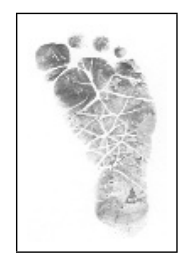

(a)

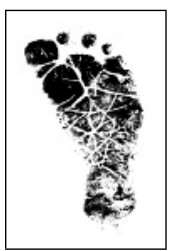

(b)

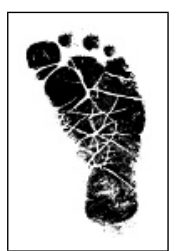

(c)

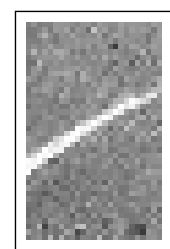

(a)

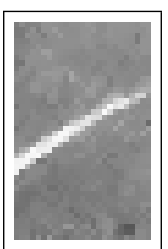

(b)

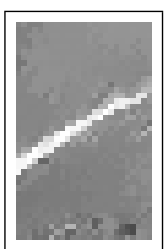

(c)

Fig. 3. Global thresholding: (a) Input image; (b) Otsu threshold=206; (c) Our method, threshold $=229$.

Fig. 4. Edge-preserving results: (a) Original image; (b) NGIW filtering; (c) Our adaptive edge-preserving filtering.

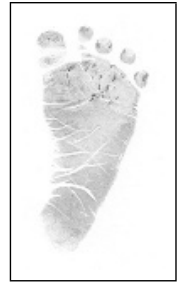

(a)

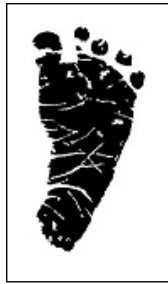

(b)

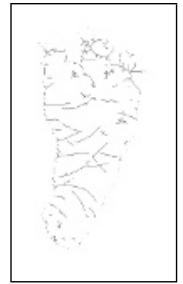

(c)

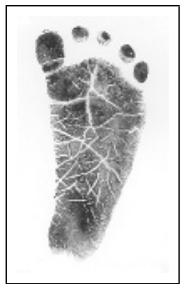

(d)

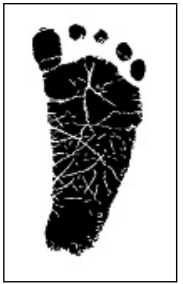

(e)

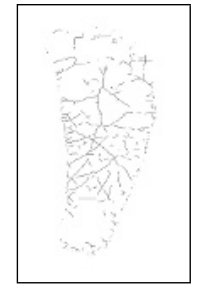

(f)

Fig. 5. Two examples of wrinkle detection and thinning: (a) and (d) are input i mages; (b) and (e) are detected wrinkles; (c) and (f) are thinned wrinkles. 
Image measurements: Measures such as length, width, area, occupation percentage in the minimum enclosing rectangle and percentage of wrinkle occupation, related to the foot and its four regions, are obtained automatically. Nevertheless, wrinkle measures, such as length, width and localization cannot be computed until the user selects an image area to be measured.

\subsection{Neonatology - Preliminary Results}

The FootScanAge method aims to analyze some characteristics of newborns' plantar surfaces and to identify its relationships with gestational age. From preliminary observations and hypotheses we see that the maturation of the newborn is accompanied by the emergence of the following characteristics:

Extremely premature newborn (EPN): Reduced size of the plantar surface, without clear delineation of the calcaneus's region (rectangle shape). Presence of plantar wrinkles, except limiting line between areas I and II, is not observed. Premature newborn (PN): Slightly larger plantar surface, beginning the delineation of the calcaneus's region. Presence of limiting lines of areas I and II, hallucal, and of areas II and III, progressively. Presence of plantar wrinkles and variable diffuse "corrugation", more accented in both areas I and II.

Term newborn (TN): Larger plantar surface, with well-defined calcaneus's region. Presence of all limiting lines of areas, wrinkles, and diffuses "corrugation".

The system was applied to a randomized sample of newborns, with 186 newborns classified according to their gestational age (in weeks): TN ( $>37)$, PN $(\leq 37)$ and EPN $(\leq 28)$. This evaluation was done to verify the viability of the system in an experimental model for adjustment of the tool and posterior application of the method. The gestational age was evaluated from four measures routinely accomplished by Neonatologists. The majority in the sample is TN (142), being the remaining PN (38) and EPN (6). The average gestational age for the sample was $38.74 \pm 2.91$ weeks. Among the TN the average was $40.09 \pm$ 0.79. For PN these values were $35.28 \pm 1.98$ and for EPN of $28.66 \pm 1.36$.

Applying the results to a model of Multiple Regression (stepwise forward), we observed that the foot length, occupation percentage in area I, and plantar surface area occupation are the main explanatory variables with a determination coefficient of $35 \%$. Applying the same model for PN, the foot area and percentage of wrinkle occupation in area I have a determination coefficient of $61.4 \%$, indicating that these variables possibly explain $62 \%$ of gestational age variability. Through Discriminant Analysis, to evaluate which variables provide the best newborn classification, the foot length, percentage of wrinkle occupation in area I, birth weight, foot width and area II width obtained a discrimination power of $97 \%$ for TN, $55 \%$ for PN and 50\% for EPN. Applying the same model for PN, the foot area and percentage of wrinkle occupation in area I obtained $97 \%$ for the PN and $50 \%$ for EPN. For all tests a minimum level significance of $5 \%$ was considered. These results confirm that the measures: length of the foot, total plantar surface area, percentage of wrinkle occupation, and the measures in the established plantar surface regions are related to the gestational age. 


\section{Conclusion}

This paper presents the main characteristics of the image-processing tool for a new method, whose goal is the identification of newborns' gestational age based on the analysis of plantar surface images. The systematic application of the developed system should bring considerable contributions for confirmation of the new theory, aiding in the extraction of significant characteristics for more precise and fast identification of gestational age in newborns. A prototype of the FootyScanAge System is being used and evaluated by experts of the UFPR University Hospital. Some hypotheses regarding the method have already been confirmed, related to both wrinkle characteristics and foot shape. Currently, we are working to add data mining (textual information about mother/baby) and image mining tools to the package to verify other important relations with respect to gestational age, not observed to date by Neonatologists. As future work, the FootScanAge System will be expanded to include child identification.

\section{References}

1. Duncan, J., Ayache, N.: Medical image analysis: Progress over two decades and the challenges ahead. IEEE Trans. on PAMI 22 (2000) 85-106

2. Sola, A., Chow, L.: The coming of (gestational) age for preterm infants. Journal of Pediatrics 135 (1999) 137-139

3. Scholl, T., Miller, L., Salmon, R., Cofsky, M., Shearer, J.: Prenatal care adequacy and the outcome of adolescent pregnancy effects on weight gain, preterm delivery, and birth weight. Obstetrics and Gynecology 69 (1987) 312-316

4. Donovan, E., Tyson, J., Ehrenkranz, R., et al.: Inaccuracy of ballard scores before 28 weeks gestation. Journal of Pediatrics 135 (1999) 147-152

5. Kilpatrick, S., Schlueter, M., Piecuch, R.: Outcome of infants born at 24-26 weeks gestation. I. survival and cost. Obstetrics and Gynecology (1997) 803-808

6. Cummins, H., C., M.: Finger prints, palms and soles: An introduction to dermatoglyphics. Dover Publications, Inc, New York (1961)

7. Olabarriaga, S., Smeulders, A.: Interaction in the segmentation of medical images: A survey. Medical Image Analysis 5 (2001) 127-142

8. Haddon, J.: Generalized threshold selection for edge detection. Pattern Recognition 21 (1988) 195-203

9. Niblack, W.: An Introduction to Digital Image Processing. Prentice Hall (1986)

10. Otsu, N.: A threshold selection method from gray-level histograms. IEEE Trans. on Systems, Man, and Cybernetics 9 (1979) 62-66

11. Serra, J.: Image Analysis and Mathematical Morphology. Academic Press (1982)

12. Adelmann, H.: An edge-sensitive noise reduction algorithm for image processing. Computers in Biology and Medicine 29 (1999) 137-145

13. Wang, X.: On the gradient inverse weighted filter. IEEE Trans. on Signal Processing 40 (1992) 482-484

14. Hung, C.C.: On the edge preserving smoothing filter. In: Proceedings of Southeastcon '97. Engineering in New Century. (1997) 12-14

15. Bellon, O., Silva, L.: New improvements to range image segmentation by edge detection. IEEE Signal Processing Letters 9 (2002) 43-45 